\title{
Um conto de três cidades: música e sensibilidades culturais urbanas
}

\section{Angela Prysthon}

\section{Resumo}

Este artigo tem por objetivo discutir as complexas e profusas articulações entre música popular e a cidade. Para isso recortamos três épocas, três estilos e três cidades diferentes: Manchester do final dos anos 70 aos anos 90; Seattle no início dos anos 90; e Recife dos anos 90 aos 2000. 0 propósito é identificar certas recorrências e diferenças entre as cenas para compreender como 0 engendramento de "sensibilidades culturais" e a configuração de cenas musicais modelam e redesenham não apenas as próprias cidades, mas o modo como os sujeitos apreendem e circulam nesses espaços.

\section{Palavras-chave}

Cidade. Música popular. Sociabilidade.

Sensibilidades culturais.

\section{Angela Prysthon | prysthon@gmail.com}

Doutora em Critical Theory And Hispanic Studies pela Universidade de Nottingham. Professora do Programa de Pós-Graduação em Comunicação da Universidade Federal de Pernambuco - UFPE.

\section{Introdução}

Este artigo tem por objetivo discutir as complexas e profusas articulações entre música popular e a cidade. Quando pensamos nessas articulações, imediatamente são feitas associações mentais entre determinados gêneros e determinadas cidades: Nova Orleans e o jazz tradicional; Nova York e o hip-hop; Chicago e o blues; Detroit e 0 som de Motown; Nashville e o country; Belém do Pará e 0 tecnobrega; 0 Rio de Janeiro e o samba; São Luís e o reggae, entre outras. Aqui vamos apresentar de modo necessariamente panorâmico a análise de três cenas distintas (no tempo e no espaço) ligadas às culturas pop e rock. São três épocas, três estilos e três cidades diferentes: Manchester, na Inglaterra, do final dos anos 70 aos anos 90; Seattle no início dos anos 90 e Recife dos anos 90 aos 2000. A idéia é identificar certas recorrências e diferenças entre as cenas para compreender como 0 engendramento de sensibilidades culturais e a configuração de cenas musicais modelam e redesenham não apenas as próprias cidades, mas o modo como os sujeitos apreendem e circulam nesses espaços. 
0 conceito de "sensibilidade cultural" empregado aqui é tributário do trabalho de Celeste

Olalquiaga sobre o pós-modernismo, no qual ela define sensibilidade "como uma predisposição coletiva para certas práticas culturais" (OLALQUIAGA, 1998, p. 16). E de fato há nos cenários observados aqui uma predisposição coletiva que construiu em torno da música um novo imaginário para as três cidades em questão. Evidentemente, esse novo imaginário não foi constituído apenas pelos produtos da indústria musical (discos, CDs, shows e presença midiática), mas por uma gama de articulações entre estes - que poderíamos classificar como "catalisadores" - e indivíduos, grupos e signos.

É necessário compreender, portanto, como se desenhou essa gama de articulações nas três cenas a partir de uma noção diferente de cidade que deixa evidente a urgência de um constante deslocamento conceitual, vinculado ao marco teórico do pós-moderno. Pois, se na modernidade tínhamos, de certa maneira, algumas convicções em relação à natureza da cidade, seus componentes, suas articulações, a partir da pósmodernidade não apenas teremos que renegociar e retrabalhar todo esse elenco de noções, como também inserir uma série de novos paradigmas e termos. Claro que sem esquecer do flâneur, do cosmopolitismo e da modernidade - elementos constitutivos do urbano que entram em cena na cidade pós-moderna de maneira muito mais enfática que antes através da descentralização, dos meios de comunicação de massa, das redes de informaç̧ão, da diferença e de novos espaços urbanos. Pois, além de um território conceitual necessariamente mais fluido, tem-se em vista uma nova materialidade. As world cities, cidades do mundo em constante processo de mutação, não são necessariamente as maiores cidades, mas lugares onde a diversidade se multiplica a cada instante, ora num movimento integrativo, ora na dissolução em partes isoladas.

As cidades mundiais são sítios nos quais encontramos a justaposição de ricos e pobres, a nova classe média de profissionais liberais e os sem-teto, e uma variedade de outras identificações étnicas, de classe e tradicionais, como também pessoas do centro e da periferia que são colocadas dentro de uma mesma localização especial. [tradução nossa] (FEATHERSTONE, 1995, p. 118)

Desse modo, a própria configuração urbana contemporânea vai sendo permeada pelo imaginário cultural e conceitual do pós-moderno. Featherstone também fala de um entrelaçamento entre as esferas cultural, social e econômica dessa cidade pós-moderna:

A cidade pós-moderna é portanto muito mais auto-consciente imagética e culturalmente; é um centro de consumo tanto cultural como geral, e assim como este último não pode ser desvinculado dos signos e imaginários culturais, os estilos de vida urbanos, o cotidiano e as atividades de lazer estão todos em maior ou menor graus influenciados pelas tendências pós-moderna simulativas (FEATHERSTONE, 1991, p. 99)

As transformações do cenário urbano mundial são quiçá lentas, graduais, mas certamente são bastante concretas. A nossa principal 
hipótese aqui é que a música (e os processos sociais ligados a ela) vai ser essencial para 0 engendramento dessas transformações.

\section{Manchester, so much to answer for}

Giacomo Bottà (2006), falando sobre a influência efetiva da música popular sobre a cidade, enumera algumas maneiras concretas através das quais é exercida essa influência:

Este é o resultado de uma superposição em camadas: a música popular media lugares como paisagens textuais, sonoras e visuais. As letras de músicas que se referem a lugares se configuram como as paisagens textuais. 0 uso da tradição musical local, vernacular ou ruídos tipicamente urbanos constituem as paisagens sonoras de uma banda. Finalmente as paisagens propriamente ditas consistem de todos os elementos visuais (por exemplo, as capas) que se referem à mesma localidade. Voltando para o nível da regeneração, parece importante notar que a música em si é etérea, mas sua produção, circulação e fruição dependem de fatores materiais localizados nas cidades. [tradução nossa] (p. 121)

Manchester é uma cidade do noroeste da Inglaterra, mais conhecida como o berço da Revolução Industrial e como uma das maiores cidades da Grã-Bretanha (a zona metropolitana de Manchester é a segunda maior aglomeração urbana do Reino Unido depois de Londres). Além das marcas da revolução industrial e da sua subseqüente decadência, o imaginário da cidade foi profundamente marcado pela Segunda
Guerra, na qual Manchester teve grande parte do seu centro histórico destruído pelas bombas. A cidade também sofreu de modo particularmente intenso as reformas econômicas da era Thatcher: indústrias fechadas, altos índices de desemprego e o fechamento do porto em 1982. 0 que afetou, obviamente, as formas de produzir e consumir cultura na cidade.

A partir do final da década de 1970 surge uma cena musical vibrante na esteira da subcultura punk em ascensão em toda a Inglaterra (subcultura, aliás, deflagrada a partir de um cenário de decadência pós-industrial extremamente semelhante ao contexto particular mancuniano $\left.{ }^{1}\right)$. Podemos ver na trajetória dessa cena (que começa com o agrupamento de bandas em artistas a partir do impulso dos primeiros shows dos Sex Pistols na cidade em 1976 e, de certo modo, tem um fechamento simbólico a partir do final da era "Madchester" com 0 encerramento das atividades do clube Haçienda em 1997) um conjunto de exemplar de modos de articulação entre música e cidade. Vários grupos - em certa altura já classificados como pós-punk -, lugares e indivíduos podem ser mencionados como parte desse período histórico tão especial para a mitologia pop: Buzzcocks, The Fall, The Smiths e Morrissey, A certain ratio, Durutti Column, The Stone Roses, Joy Division e Ian Curtis, New Order, Happy Mondays, Factory Records (gravadora), Haçienda (clube 
noturno aberto em 1982), Tony Wilson (jornalista e empresário), Rob Gretton (empresário de bandas), Peter Saville (designer gráfico).

Os fragmentos urbanos indubitavelmente permeiam a história musical da cidade. Por exemplo, nas letras de Morrissey, vocalista da banda extinta em 1986 The Smiths, as paisagens de Manchester estão sempre presentes. Desde a alusão a trens, pontes, fontes, cemitérios e escolas, até crimes, filmes e livros que tematizam a cidade. Como na canção Suffer Little Children (1983), que alude aos assassinatos em série de crianças perpetrados por Myra Hindley e Ian Brady nos anos 60 e na qual Morrissey vai mencionando os nomes das vítimas:

\section{Lesley-Anne, with your pretty white beads Oh John, you'll never be a man And you'll never see your home again Oh Manchester, so much to answer for ${ }^{1}$}

Outro exemplo direto da presença de Manchester no cancioneiro dos Smiths é The Headmaster Ritual (1984), na qual é descrita a rotina numa escola da cidade:

Belligerent ghouls
Run Manchester schools
Spineless swines
Cemented minds ${ }^{3}$

Já Reel around the fountain evoca uma representação mediatizada de Manchester ao citar diretamente frases do filme A Taste of Honey (Tony Richardson, 1962), ambientado em Manchester e uma constante referência para 0 grupo, em capas, vídeos e letras:

Reel around the fountain
Slap me on the patio
I'll take it now
Oh ...
Fifteen minutes with you
Well, I wouldn't say no
People said that you were virtually dead
And they were so wrong

Entretanto, de todas as figuras individuais da cidade, a que talvez sintetize melhor esse período da história da música pop de Manchester seja Tony Wilson, o empresário e jornalista que impulsionou a "movida mancuniana", desde os primeiros shows punk até a cristalização de Madchester (como Manchester começou a ser chamada a partir do final dos anos 80 e da configuração da cena acid e techno na cidade). Em meados dos 70, Wilson era um repórter de TV local com o que ele chamava de 'excesso de orgulho cívico' e grandes planos para a cultura do noroeste da Inglaterra (24 Hour Party People): ocupando o lugar central nesses planos estava a Factory Records, a gravadora que de certo modo

"Lesley-Anne, com seu bonito colar de bolinhas brancas/ Oh, John, você nunca será um homem/ E você nunca verá sua casa novamente/ Oh, Manchester, tanto para dar conta". [tradução nossa]

"Zumbis beligerantes/ Dirigem as escolas de Manchester/ Suínos invertebrados/ Mentes cimentadas". [tradução nossa]

"Carretel em volta da fonte/Estapeia-me no pátio/ Tomarei agora/ Oh.../ Quinze minutos com você/ Eu não recusaria/ As pessoas disseram que você estava virtualmente morto/Mas eles estavam tão errados. [tradução nossa] 
estabeleceu novos parâmetros para o lançamento e a circulação do rock e da música pop no mundo. Nem todos os artistas e grupos importantes da cena mancuniana faziam parte da Factory, mas a relação da gravadora com a cidade foi tão intensa que deixou marcas muito fortes mesmo após a falência em 1992. 0 selo, lançado em 1978, empregava um sistema de catalogação inusitado no qual não apenas os lançamentos musicais, mas também os trabalhos gráficos, edifícios e outros objetos recebiam um número. Até 0 caixão no qual foi enterrado Wilson em agosto de 2007 recebeu um número de catálogo: FAC 501. 0 caso da Factory sintetiza todas as possibilidades de relação entre música e cidade. Aliás, a Factory surge das entranhas de Manchester, é um projeto completamente desvinculável da cidade, em todos os seus aspectos (visuais, sonoros, líricos).

Um dos exemplos mais notáveis dessa simbiose, especialmente relativa aos "soundscapes" urbanos são as gravações do grupo Joy Division, especialmente aquelas produzidas por Martin Hamnett, nas quais aparecem ruídos de trens, alarmes, maquinaria pesada, a acústica dos grandes armazéns, entre outros efeitos (OTT, 2003). Nas letras das canções de vários dos grupos da Factory, inclusive do Joy Division, também é possível vislumbrar algumas referências mais indiretas, especialmente a uma atmosfera lúgubre e sombria que remete a certa decadência pósindustrial. Assim como nas letras dos Happy Mondays, evocativas da psicodelia e do ecstasy massivamente consumidos em Madchester. Mas talvez seja nos landscapes propriamente ditos que a conexão entre cidade e música feita pela Factory seja mais explícita ou bem sucedida: não somente 0 clube Haçienda se tornou uma espécie de símbolo cultural mor da cidade durante a sua existência, como no trabalho gráfico que marcou a gravadora (especialmente aquele empreendido pelo designer Peter Saville).

\section{Smells like teen spirit}

0 caso de Seattle difere do de Manchester pela ausência de referências tão explícitas. Não é possível fazer a mesma taxonomia de letras, capas e sons do grunge que tenham rastros tão plásticos da cidade. Entretanto, é provável que o impacto da cena grunge em Seattle tenha sido ainda maior (até pensarmos nos termos da influência internacional que teve o movimento). 0 grunge é um estilo de rock alternativo surgido na segunda metade dos anos 80 no estado de Washington nos Estados Unidos, especialmente na área de Seattle. As influências do punk, do heavy metal e do hardcore aliadas a uma estética visual despojada, letras que versavam principalmente sobre a apatia e a angústia da chamada "geração X" (pessoas que estavam na casa dos vinte anos na década de 90) e uma rejeição do glamour e da performance estilizada que caracterizou o rock e o pop dos anos 80 .

Assim como em Manchester, um selo em particular vai ter preponderância na cena: a gravadora Sub Pop, que concentrou grande parte das bandas de Seattle e catapultou os 
dois grandes nomes do grunge para o sucesso mundial através daqueles que que talvez sejam os dois álbuns mais "clássicos" da cena: Nirvana, com Nevermind, e Pearl Jam, com Ten, ambos lançados no segundo semestre de 1991. Além do Nirvana e do Pearl Jam, outras bandas chegaram ao estrelato (nem todas vinculadas ao Sub Pop) do mesmo modo ambíguo e indeciso - um misto de atração e repulsa em relação à indústria fonográfica e ao showbiz: Alice in Chains, Soundgarden, Mudhoney, Green River, Melvins, entre outras de menor expressão.

Outro aspecto que chama a atenção é como, de um modo até mais intenso que Manchester - que tinha algo de misoginia -, a cena de Seattle é emblemática da predominância masculina nos seus grupos musicais e na própria configuração das redes sociais ligadas a ela. Algo que pode ser vagamente relacionado ao boom da tecnologia na década de 90 com a chegada massiva de homens solteiros na região para trabalhar em empresas como Microsoft, por exemplo. A cena se configurou quase como uma catarse tanto para os jovens que faziam a música, como para aqueles que a consumiam.

Contudo, o mais relevante da cena grunge em conexão com a cidade de Seattle é simultaneamente o modo como ela foi transformada pelos símbolos (ou poderíamos dizer anti-símbolos) associados à música (sobretudo a moda - as camisas de flanela, os cabelos sujos e desgrenhados, os coturnos - e 0 comportamento anti-establishment dos músicos) e associada ao grunge aos olhos do mundo. Para além de sua fama como um concentrado de empresas tecnológicas (especialmente aquelas ligadas à informática), como o berço da Starbucks e dos cafés de designer, mas de certo modo associado a tudo isso, Seattle foi se tornando sinônimo do grunge.

Começou-se a prestar atenção naquela isolada e fria cidade do noroeste americano a partir de uma cena que pouco tinha de hedonista e afirmativa. Mas a interessante contradição é que tudo o que o movimento tinha de negação (rejeição do mainstream, do padrão, antiesteticismo, antiindústria) foi sendo capitalizado para a caracterização de Seattle como um dos pólos criativos mundiais de maior impacto e relevância na década de 1990.

Curiosamente, o vigor do grunge só foi possível por causa do relativo isolamento cultural vivido pela cidade até então:

De acordo com aqueles que estiveram lá, Seattle era um lugar bem isolado culturalmente. As grandes bandas sequer incluíam Seattle nas turnês americanas da Costa Oeste, e a cena local estava repleta bandas derivativas que faziam o máximo para soar como outras. Não era um ambiente que anunciasse uma explosão de vitalidade musical original. Ainda assim, 0 ambiente parece ser um conceito-chave para explicar a década de 1985-1995. (HOWITT) [documento eletrônico]

Ou seja, a cena surge exatamente de uma carência, aparece como resposta a um contexto 
francamente fechado e provinciano. A cidade se torna plataforma para a elaboração de estratégias de superação deste contexto, ela é a malha através das quais as predisposições coletivas são articuladas.

\section{A cidade não pára}

Em vários trabalhos sobre a cena Mangue foi apontada a ligação do movimento com a cidade, com a cultura urbana e com a emergência de novas identidades sociais na periferia. Emergindo da "periferia da periferia", da lama, o mangue bit (como foi chamado no início pelos grupos que o constituíam) ou mangue beat (como ficou conhecido através da mídia nacional) vai transformar a cidade do Recife. Assim como Manchester e Seattle, a perspectiva de transformação urbana através da música, da cultura, é o motor das sensibilidades culturais.

Nos trabalhos das bandas manguebit (mesmo aquelas que rejeitam o rótulo, eventualmente) estão presentes tanto a rearticulação da tradição (através de ritmos populares de Pernambuco e alusões ao folclore da região), como a preocupação com as últimas tendências da cultura pop mundial. Recuperando 0 elo perdido (e certa independência vital e muitas vezes franca oposição) em relação ao tropicalismo, Chico Science \& Nação Zumbi, por exemplo, em Da lama ao caos mistura ritmos brasileiros como o maracatu, a ciranda ou o coco com o samba, com música eletrônica, hip hop e rock. As letras do Nação Zumbi freqüentemente tentam essa equação entre o local (as especificidades de viver numa cidade particularmente subdesenvolvida de um país subdesenvolvido, as gírias e os mitos recifenses) e 0 universal (as relações com a tecnologia, as imagens metropolitanas). As canções mais conhecidas do grupo tematizam justamente 0 "inchaço" do Recife, a sujeira e, simultaneamente, a música de suas ruas.

0 diálogo entre as dualidades tradição/ modernidade, centro/periferia, nacionalismo/ cosmopolitismo vai ser explorado nos trabalhos seguintes, por exemplo, no segundo disco, inclusive quando vai ser indiretamente assumida certa herança do Tropicalismo com a participação especial de Gilberto Gil na faixa Macô e a regravação de Maracatu Atômico de Jorge Mautner. Em Enquanto o mundo explode, Science afirma:

$$
\begin{aligned}
& \text { um curupira já tem seu tênis importado } \\
& \text { não conseguimos acompanhar o motor } \\
& \text { da história } \\
& \text { mas somos batizados pelo batuque e } \\
& \text { apreciamos agricultura celeste. (1996) }
\end{aligned}
$$

0 outro grupo mais proeminente do mangue beat, o mundo livre s/a, embora ritmicamente mais convencional que o Nação Zumbi, reunindo algumas características do samba e do rock, procura explicitar a posição da periferia em relação ao mundo globalizado. Recife continua sendo referência importante como perspectiva periférica: 
0 mangue reanima, abastece

Injeta, recarrega as baterias

Da Veneza esclerosada

Mangue, manguedown

Cidade complexo, caos portuário

Mangue, Manguetown. (1994)

Outro dado importante na conjuntura do manguebit é, indubitavelmente, como o discurso da identidade e da tradição ultrapassou as barreiras da cultura das classes médias e letradas para influenciar a produção musical mais popular. Vemos, assim, a emergência de artistas realmente periféricos (periféricos dentro da própria periferia) fazendo uso do discurso da identidade nacional e de suas relações com formas globais de expressão. Mesmo podendo ser considerado mais culto que outros fenômenos mais populares (o hip-hop de São Paulo, o funk carioca e o pagode, por exemplo) por suas características ideológicas, discursivas e metalingüísticas, lança alguns dados importantes para o redimensionamento do papel do popular no contexto contemporâneo: se no início eram bandas urbanas lideradas por filhos da classe média (mesmo que em alguns grupos houvesse a presença de membros das classes mais baixas) a ganharem expressão nacional, nos últimos anos da década de 90 foram mercantilizados sob esse rótulo (às vezes até inadequadamente) artistas de origem indiscutivelmente "popular" e proletária, como Selma do Coco (uma senhora de idade "revelada" ao público num festival de rock em Recife em 1996), a cirandeira Lia de Itamaracá ou as bandas hardcore do subúrbio Alto José do
Pinho, também de Recife. Todos conquistaram espaço na mídia, desde programas de auditório de grande audiência a documentários na MTV ou na TV Cultura.

Contudo, é provável que a grande contribuição do Mangue seja realmente a interferência na cultura da cidade. Um dos aspectos mais relevantes da história do movimento é precisamente essa relação com o Recife, ou, melhor ainda, a maneira como seus produtos, manifestações, modos e modas foram construindo ao longo da década de 90 uma nova relação com a cidade, uma nova cultura urbana. Em várias "genealogias" do Mangue se aponta a influência do Recife (e quase sempre a influência da pobreza, da miséria e das mazelas da cidade) nas letras, nas músicas, no visual e na atitude dos músicos, onde talvez o ponto mais interessante seja a forma como todos esses elementos acabaram por transformar 0 imaginário urbano recifense, a maneira como o Mangue construiu uma política de diferença cultural para a cidade, o modo como, através dos mais variados fenômenos culturais, 0 Recife se viu repentinamente inserido num contexto pósmoderno. 0 Recife foi, pois, reinventado a partir do movimento Mangue, ou melhor, da "cena Mangue", como preferem seus "fundadores".

\section{Convergências periféricas}

As três cenas são evidentemente distintas, são espacialmente muito distantes umas das outras, mesmo que temporalmente haja coincidências. 
Contudo, chama a atenção como recorrência 0 modo a partir do qual as sensibilidades culturais aparecem como constitutivas do tecido urbano, como tais articulações (tanto a música propriamente dita, como todo o seu entorno, seus acessórios - moda, audiovisual, códigos de comportamento, etc.) se tornam as bases para a inserção (ou reinserção) dessas cidades num contexto globalizado. Manchester, Seattle, Recife, em espaços-tempos distintos e cada uma de sua maneira particular, demonstram 0 funcionamento do que poderíamos chamar de cosmopolitismo pós-moderno ou cosmopolitismo periférico (PRYSTHON, 2002), processo sublinhado e condicionado por uma série de remapeamentos culturais implicados na globalização e numa reconfiguração pósmoderna do conceito de cidade.

As sensibilidades culturais das três cenas apontam justamente para o momento de ruptura representado pelo pós-modernismo para a cultura das cidades. Elas são exemplos bastante concretos de como o pós-modernismo e a pós-modernidade têm relações, ou antes, podem ser conseqüências da política mundial contemporânea e de uma completamente nova configuração global de poder, "in which the old imperial maps have been lost" (na qual os velhos mapas imperiais se perderam) - como faz Robert Young (1990, p. 117). Ou seja, poderíamos pensar no momento de ruptura do pós-moderno como o momento de autoconsciência cultural da periferia (e entendendo essas cidades "fora do eixo" - seja Manchester, Seattle ou Recife - como encarnações urbanas do conceito de periferia). 0 cosmopolitismo vai-se reconfigurando através do percurso de autodescoberta feito pelas margens. Uma autodescoberta que pode levar ao estabelecimento das primeiras políticas da diferença e para a afirmação de um novo conceito de urbano. 0 cosmopolita periférico tenta se colocar, produzir e se autodefinir a partir de uma instância ambígua (ser e estar na periferia, desejar estar na metrópole, no centro) e aponta justamente os elementos que fazem da periferia um modelo de modernidade alternativa (problemática, incompleta, contraditória). Ele trabalha nos interstícios de uma realidade e tradições locais e de uma cultura urbana internacional, aspiracional e moderna. Temos assim outro cosmopolitismo que indubitavelmente transforma a própria noção de cidade, de experiência urbana na contemporaneidade.

As teorias pós-modernas e do pós-moderno, inevitavelmente, pois, lançam outras dimensões ao conceito de cosmopolitismo: a sua constante remissão ao crescente descentramento da vida urbana e da cultura pós-moderna, a evidente globalização em diversas esferas da sociedade - entre elas economia e cultura -, a insistência pelo relativismo cultural e o estabelecimento de um ciberespaço agora como realidade e não mais alucinação futurista são algumas das razões mais importantes para essa redefinição do cosmopolitismo. Basicamente, entretanto, 
a emergência dessa sociedade pós-industrial, ou "sociedade de informação"- com todas as suas nuances, entre elas a valorização do periférico, do exótico, do excêntrico (refletidos no multiculturalismo) - desesestabiliza a força centralizadora das metrópoles modernas. 0 cosmopolitismo pós-moderno e periférico vai ser diferente, sobretudo porque ele não supõe necessariamente um ponto norteador (algo essencial no cosmopolitismo moderno com a Paris-mito dos modernos e os subseqüentes prolongamentos dessa Paris na periferia - São Paulo, Buenos Aires, etc.).

Portanto, se o cosmopolitismo moderno é essencialmente centrípeto, a força centrífuga da pós-modernidade começa a relativizar a importância das grandes metrópoles mundiais em termos de disseminação das informações. 0 que antes era quase um sistema de oposições campo/cidade; provinciano/cosmopolita; bárbarie/ civilização; caos/ordem -, torna-se uma rede de múltiplas interdependências, confluências e novos parâmetros. E é justamente a cidade que se torna 0 território intersticial onde se encadeiam, intercalam-se e se confrontam tais oposições. Ao invés de ser apenas mais um elemento do binarismo oposicional, a cidade passa a ser ela própria um processo dialético dos embates pós-modernos.

0 que não significa, obviamente, que deixem de existir os grandes centros de onde emanam as tendências culturais. Porém como Manchester,
Seattle e Recife demonstraram nas três últimas décadas, há uma clara propensão para que essas tendências apareçam de muitos outros lugares, difundam-se e dissolvam-se de forma muito mais rápida. A gradual superação desses esquemas oposicionais e a crescente descentralização cultural da contemporaneidade vão, assim, modificando profundamente a própria estrutura da teorização sobre a cidade e nossas próprias experiências.

\section{Referências Bibliográficas}

BOTTÀ, Giacomo. Pop music, cultural sensibilities and places: Manchester 1976-1997. In: FORNÄS, Johan (ed). THE ESF-LIU CONFERENCE CITIES AND MEDIA: cultural perspectives on urban identities in a mediatized world, 2006, Vadstena, Suécia. Disponível em: <http://www.ep.liu.se/> Acesso em: 3 mar. 2008.

\section{FEATHERSTONE, Mike. Consumer culture and} postmodernism. Londres: Sage, 1991.

Undoing culture: globalization, postmodernism and identity. Londres: Sage, 1995.

HOWITT, Bernie. Popular culture- grunge. In: Society and culture Association. Disponível em: <http://www. ptc.nsw.edu.au/scansw/grunge.html>. Acesso em: 3 mar. 2008.

KING, Emily (ed). Designed by Peter Saville. Nova York: Princeton Architectural Press, 2003.

OLALQUIAGA, Celeste. Megalópolis: sensibilidades culturais contemporâneas. São Paulo: Studio Nobel, 1998. OTT, Chris. Joy Division's unknown pleasures. Londres: Continuum, 2003.

\section{PRYSTHON, Angela. Cosmopolitismos periféricos:} ensaios sobre modernidade, pós-modernidade e Estudos Culturais na América Latina. Recife: Bagaço, 2002. 
REYNOLDS, Simon. Beijar o céu. São Paulo: Conrad, 2006.

ROBERTSON, Matthew. Factory records: the

complete graphic album. San Francisco: Chronicle

Books, 2007.

WILSON, Tony. Twenty four hour party people: what the sleeve notes never tell you. Londres: Channel 4

Books, 2002.

YOUNG, Robert. White Mythologies. Writing History

and the West. Londres: Routledge, 1990. 
A tale of three cities: music and urban cultural sensibilities

\section{Un cuento de tres ciudades: música y sensibilidades culturales urbanas}

\section{Abstract}

This article aims to discuss the complex and profuse articulations between popular music and the city. We chose as objects three different cities, styles and historical periods: Manchester from the end of the 1970s to the beginning of the 1990s; Seattle at the beginning of the 1990s and Recife from the 1990s to the 2000s. Our purpose is to identify certain recurrences and differences between these scenes to understand how cultural sensibilities are established and how musical movements model and redesign not only the actual cities, but also the way in which individuals apprehend and circulate in theses spaces.

\section{Keywords}

City. Popular music. Sociability.

Cultural sensibilities.

\section{Resumen}

Este artículo tiene por objetivo discutir las complejas y profusas articulaciones entre la música popular y la ciudad. Para esto elegimos tres épocas, tres estilos y tres ciudades distintas: Manchester desde el final de los años 70 hasta los años 90; Seattle en principios de los 90 y Recife de los años 90 a los 2000 . El propósito es identificar recurrencias y diferencias entre las escenas para comprender cómo las sensibilidades culturales son engendradas y cómo las escenas musicales modelan y rediseñan no sólo las propias ciudades, pero la manera cómo los sujetos aprehenden y circulan en esos espacios.

\section{Palabras clave}

Ciudad. Música popular. Sociabilidad. Sensibilidades culturales. 


\section{Expediente}

A revista E-Compós é a publicação científica em formato eletrônico da Associação Nacional dos Programas de Pós-Graduação em Comunicação (Compós). Lançada em 2004, tem como principal finalidade difundir a produção acadêmica de pesquisadores da área de Comunicação, inseridos em instituições do Brasil e do exterior.
E-COMPÓS I www.e-compos.org.br I E-ISSN 1808-2599

Revista da Associação Nacional dos Programas de Pós-Graduação em Comunicação. Brasília, v.11, n.1, jan./abr. 2008.

A identificação das edições, a partir de 2008, passa a ser volume anual com três números.

\section{CONSELHO EDITORIAL}

\section{Afonso Albuquerque}

Universidade Federal Fluminense, Brasil

Alberto Carlos Augusto Klein

Universidade Estadual de Londrina, Brasi

Alex Fernando Teixeira Primo

Universidade Federal do Rio Grande do Sul, Brasi

Alfredo Vizeu

Universidade Federal de Pernambuco, Brasil

Ana Carolina Damboriarena Escosteguy

Pontifícia Universidade Católica do Rio Grande do Sul, Brasil

Ana Silvia Lopes Davi Médola

Universidade Estadual Paulista, Brasil

André Luiz Martins Lemos

Universidade Federal da Bahia, Brasil

Ângela Freire Prysthon

Universidade Federal de Pernambuco, Brasil

Antônio Fausto Neto

Universidade do Vale do Rio dos Sinos, Brasil

Antonio Carlos Hohlfeldt

Pontifícia Universidade Católica do Rio Grande do Sul, Brasil

Arlindo Ribeiro Machado

Universidade de São Paulo, Brasil

César Geraldo Guimarães

Universidade Federal de Minas Gerais, Brasi

Cristiane Freitas Gutfreind

Pontifícia Universidade Católica do Rio Grande do Sul, Brasil

Denilson Lopes

Universidade Federal do Rio de Janeiro, Brasil

Eduardo Peñuela Cañizal

Universidade Paulista, Brasil

Erick Felinto de Oliveira

Universidade do Estado do Rio de Janeiro, Brasil

Francisco Menezes Martins

Universidade Tuiuti do Paraná, Brasil

Gelson Santana

Universidade Anhembi/Morumbi, Brasil

Hector Ospina

Universidad de Manizales, Colômbia

leda Tucherman

Universidade Federal do Rio de Janeiro, Brasil

Itania Maria Mota Gomes

Universidade Federal da Bahia, Brasil

Janice Caiafa

Universidade Federal do Rio de Janeiro, Brasil

Jeder Silveira Janotti Junior

Universidade Federal da Bahia, Brasil
John DH Downing

University of Texas at Austin, Estados Unidos

José Luiz Aidar Prado

Pontifícia Universidade Católica de São Paulo, Brasil

José Luiz Warren Jardim Gomes Braga

Universidade do Vale do Rio dos Sinos, Brasi

Juremir Machado da Silva

Pontifícia Universidade Católica do Rio Grande do Sul, Brasil

Lorraine Leu

University of Bristol, Grã-Bretanha

Luiz Claudio Martino

Universidade de Brasília, Brasil

Maria Immacolata Vassallo de Lopes

Universidade de São Paulo, Brasil

Maria Lucia Santaella

Pontifícia Universidade Católica de São Paulo, Brasil

Mauro Pereira Porto

Tulane University, Estados Unidos

Muniz Sodre de Araujo Cabral

Universidade Federal do Rio de Janeiro, Brasil

Nilda Aparecida Jacks

Universidade Federal do Rio Grande do Sul, Brasil

Paulo Roberto Gibaldi Vaz

Universidade Federal do Rio de Janeiro, Brasil

Renato Cordeiro Gomes

Pontifícia Universidade Católica do Rio de Janeiro, Brasil

Ronaldo George Helal

Universidade do Estado do Rio de Janeiro, Brasil

Rosana de Lima Soares

Universidade de São Paulo, Brasil

Rossana Reguillo

Instituto Tecnológico y de Estudios Superiores do Occidente, México

Rousiley Celi Moreira Maia

Universidade Federal de Minas Gerais, Brasil

Sebastião Carlos de Morais Squirra

Universidade Metodista de São Paulo, Brasil

Simone Maria Andrade Pereira de Sá

Universidade Federal Fluminense, Brasil

Suzete Venturelli

Universidade de Brasília, Brasil

Valério Cruz Brittos

Universidade do Vale do Rio dos Sinos, Brasil

Veneza Mayora Ronsini

Universidade Federal de Santa Maria, Brasil

Vera Regina Veiga França

Universidade Federal de Minas Gerais, Brasil

\section{COMISSÃO EDITORIAL}

Ana Gruszynski I Universidade Federal do Rio Grande do Sul, Brasil João Freire Filho I Universidade Federal do Rio de Janeiro, Brasil Rose Melo Rocha I Escola Superior de Propaganda e Marketing, Brasil

\section{CONSULTORES AD HOC}

Bianca Freire-Medeiros I Fundação Getulio Vargas, Brasil Josimey Costa da Silva I Universidade Federal do Rio Grande do Norte, Brasil Maria Conceição Golobovante I Pontifícia Universidade Católica de São Paulo, Brasil Marlyvan Moraes de Alencar I Centro Universitário SENAC-SP, Brasil Miriam de Souza Rossini I Universidade Federal do Rio Grande do Sul, Brasil Paulo Ribeiro I Pontifícia Universidade Católica do Rio de Janeiro, Brasil Rita Alves de Oliveira I Centro Universitário SENAC, Brasil

REVISÃO DE TEXTO E TRADUÇÃO I Everton Cardoso ASSISTÊNCIA EDITORIAL E EDITORAÇÃO ELETRÔNICA I Raquel Castedo
COMPóS I www.compos.org.br

Associação Nacional dos Programas de Pós-Graduação em Comunicação

Presidente

Erick Felinto de Oliveira

Universidade do Estado do Rio de Janeiro, Brasil erickfelinto@uol.com.br

Vice-presidente

Ana Silvia Lopes Davi Médola

Universidade Estadual Paulista, Brasil

asilvia@faac.unesp.br

Secretária-Geral

Denize Correa Araújo

Universidade Tuiuti do Paraná, Brasil

denizearaujo@hotmail.com 